\title{
Common Uropathogens That Cause Utis In Enugu Area And Their Sensitivity To Ciprofloxacin
}

\author{
Onyemelukwe, $\mathrm{NF}^{1}$ and Nwokocha, $\mathrm{ARC}^{2}$ \\ ${ }^{I}$ Department of Microbiology, College of Medicine, University of Nigeria Enugu Campus, Enugu \\ ${ }^{2}$ Department of Paediatrics i/c Adolescent Health, College of Medicine, University of Nigeria Enugu Campus, Enugu
}

\begin{abstract}
Introduction: The study aims at assessing the common bacterial agents causing urinary tract infections (UTI) in Enugu and environs and to examine the incidence of Ciprofloxacin resistance in the strains of bacteria isolated from patients suspected to have UTI over an 18 month period.

Methods: Ciprofloxacin was evaluated along with other commonly used antibiotics against a total of 147 bacterial isolates obtained in pure cultures from urine samples. The patients were all out-patients seen attending urban and rural health centres in Enugu and environs with signs and symptoms suggestive of UTI. The antibiogram was done using the disk difference method.

Results: Among the tested antibiotics, high resistance was recorded for Ciprofloxacin for almost all isolates Staph saprophyticus (30.6\%), Pseudomonas aereginosa (25\%), Proteus spp (25\%), Klebsiella spp (22.9\%), Enterobacter (18.3\%), E. coli (16.1\%) and Enterococcus faecalis (15\%). A general trend which was even higher was recorded for most other tested drugs for the UTI isolates.

Conclusion: The study suggests an emergence of resistance of Ciprofloxacin among UTI pathogens and for practically other antibiotics. The worrisome increased resistance against Ciprofloxacin must demand a more serious and coordinated scrutiny and monitoring of the quality of these drugs and their rational use.
\end{abstract}

Keywords: antibiotics, resistance, urinary tract infections (UTI), Ciprofloxacin.

\section{Introduction}

Urinary tract infections (UTI) are considered to be one of the most common bacterial infections ${ }^{1}$. Accurately assessing the incidence of UTIs is difficult because they are not reportable diseases. Uropathogens are pathological organisms that cause UTI. Among all UTI organisms, Eschericha coli (E. coli) accounts for up to $90 \%$ while Staphylococcus saprophyticus accounts for up to $15 \%$ of cases of uncomplicated cystitis in most parts of the world ${ }^{2}$.

With the growing number of emerging uropathogens and the simultaneous increase of newer antibiotics, it is mandatory that laboratories use standardized methods and report only appropriate antibiotics for UTIs. Historically, one of the primary functions of the clinical microbiology laboratory has been to measure antibiotic resistance trends. Today, this is even more important and requires 'focused' antibiograms that factor these historical resistance fingerprints to selected patient location in patient versus out-patient status, disease types and age. Most importantly, these should be evaluated more frequently and should formulate to help clinicians choose empirical therapy especially for the youth who abhor hospital visits whenever they can.

From previous results elsewhere ${ }^{1-4}$, fluoroquinolones are preferred as initial agents for empiric therapy of UTI in areas where resistance is likely to be of concern. Studies have shown that they have high bacteriological and clinical cure rates as well as low rates of resistance, among most uropathogens ${ }^{4}$. Because of its availability in oral and intravenous formulations, Ciprofloxacin is the most frequently prescribed fluoroquinolone for UTIs. The drug is well absorbed orally, also rapidly excreted also rapidly excreted from the body under normal conditions and has an excellent activity against common uropathogens ${ }^{3,5}$.

However, reprints from different parts of the world have shown increasing resistance to fluoroquinolones like Ciprofloxacin. Such studies include those from Spain, China and Palestine ${ }^{6-8}$ In Nigeria, like in many developing nations of the world, drugs are usually bought and consumed without prescription, or inadequately consumed due to lack of money for the full dose. There is therefore a pressing need to assess our local empirical choices for managing UTI in this part of Nigeria.

The present study therefore attempts to describe the most common bacterial pathogens in Enugu, Nigeria as well as evaluating the anti bacterial activity of Ciprofloxacin against the common bacterial agents causing UTI in the area amongst out-patients.

\section{Methods}

A total of 650 clean voided mid-stream urine samples were collected from patients aged between 16 and 65years. During the period, June 2010 and December 2011, 385 females and 265 males were seen or referred from 3 health centers in Enugu and environs - Abakpa Health Center, Ama-oji Nike Health Centre, 
Polyclinic Asata, Enugu - and the Emmanuel Clinic, Uwani, Enugu. The study was carried out at the Emmanuel Research Centre and the Department of Medical Laboratory Sciences of the University of Nigeria Enugu Campus.

One sample per patient was collected from each of the subjects suspected to have UTI, with borate bottles (Universal containers - Sterilin UK). Each subject was advised on how to collect the samples. The nature of the study was fully explained to them and the study was conducted with their informed consent. Each specimen was inoculated on to blood agar and Mc Conkey agar plates using $0.01 \mathrm{ml}$ standard wire loop for semi quantitative counts and incubated aerobically for $24-48 \mathrm{hr}$ at $37^{\circ \mathrm{C}}$. The number of the colonies (pure growths) was counted after the incubation period. Significant culture was taken as the growth of a single colonial type (micro-organism) at a concentration of $\geq 10^{5} \mathrm{CFU} / \mathrm{ml}$ of urine.

Significant growth was identified using biochemical and serological methods after gram staining using standard bacteriological methods 9 . All gram negative rods were identified using the IMViC tests (indole, methyl red, Voges - pransker and citrate tests). Gram positive cocci were identified using catalase, coagulase, novobiocin disc sensitivity, DNase and Staph Latex tests. Characterisation was also done on the isolates by observing colonial morphology and noting haemolysis on blood Agar plates. Enterococci were also confirmed by the bile aesculin tests and serologically using 'Strep - Check test' (Lorne laboratories Ltd).

\section{Antimicrobial Sensitivity Tests}

Antimicrobial sensitivity testing of all isolates was performed on sensitivity test plates using the Kerby Baner method ${ }^{10}$. Bacteria were inoculated in $4-5 \mathrm{ml}$ sterile normal saline adjusted to a 0.5 Mcfarland Standard. A sterile cotton wool swab was used to streak the surface of Mueller Hinton Agar plates and blood agar plates ensuring the entire smooth uniform coverage of the plates. Filter paper disks impregnated with known concentrations of the antimicrobial drugs obtained commercially (Polytex) were used. The antimicrobial agents tested were: Nitrofurantoin-300ug, Gentamicin-10ug, amikacin-30ug, Nalidixic acid-30ug, Ciprofloxacin-5ug, Cotrimoxazole-1.25-23.75ug, Cephtazidine-30ug, Cefuroxime-30ug, Cephlexin-30ug, Amoxycillin-25ug.

Quality controls were included using standard strains of E. coli and Pseudomonas aeruginosa.

Statistical analysis was performed by Chi Square and P-values of, 0.05 .

\section{Results}

Of the 650 urine samples processed, 147 (22.6\%) showed pure culture of a single bacterial growth each. Gram negative bacteria yielded $109(74.1 \%)$ of the positive cultures while Gram positive bacteria yielded $38(25.9 \%)$. Of the 385 females sampled, $118(80.3 \%)$ were positive while $29(19.7 \%)$ were positive amongst the 265 males for the total 147 bacterial isolates encountered in the study.

A summary of the different bacterial agents encountered in the study is shown in Table I. Eschericha $\underline{\text { coli } i}$ is the predominant uropathogen causing UTI in the study population (42.2\%) followed by Staphylococcus saprophyticus $(21.8 \%)$ while Enterococcus faecalis $(4.1 \%)$ is the least encountered.

The isolated bacteria showed wide differences in their susceptibility to the tested antimicrobial drugs. All the bacterial isolates showed very high resistance rates to Cotrimoxazole, Nalidixic acid and Amoxycillin. Other drugs like Cephalexin, Nitrofuratoin and Gentamycin were also resisted by most of the isolates (Table 2).

A high resistance rate to Ceprofloxacin was observed for Staphylococcus saprophyticus (30.6\%), followed by Proteus mirabilis and Pseudomonas aeruginosa each 25\%, Klebsiella pneumonia- 22.9\%, Enterobacter spp (18.3\%) while E. coli was $16.1 \%$ and Enterococcus faecalis, $15 \%$ were the least resistant.

\section{Discussion}

The current work has attempted to describe the most common bacterial cause of UTI among outpatients in Enugu area of south eastern Nigeria. In the study, the predominance of $E$. coli was similar to any previous studies all over the world ${ }^{11}$. The second place in the preponderance of the uropathogens was occupied by Staphylococcus saprophyticus. Studies world-wide have shown that this organism is responsible for symptomatic UTI in sexually active women younger than 40 years of age who are otherwise healthy ${ }^{12}$, and

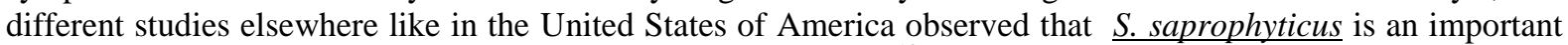
UTI pathogen for young female patients (youths)(Johnson et al) ${ }^{13}$.

Rupp ${ }^{14}$ and his group, working in similar age in Virginia, USA, found that $6.9 \%$ women were found to be colonized by $\underline{S \text {. saprophyticus. }}$.

The current study is not surprising where $29 \%$ of women in the age group 16 up to 35years were infected by $\underline{S}$ saprophyticus.

Further studies documenting the importance of $\underline{S}$. saprophyticus as a cause of cystitis in young females abound world-wide ${ }^{15,16}$. Comparison among different studies concerning resistance of uropathogens to different antimicrobial agents should take into account the different periods as well as the different socioeconomic and socio-epidemiological and chemical parameters of the target population. This can vary from country to country. 
The resistance of the drugs tested showed high resistance rates in Amoxycillin, Nalidixic acid and Cotrimoxazole and to an extent to Cephalexin, Ciprofloxacin, Nitrofurantoin and Gentamicin while the lowest was Ceftazidine and to an extent Cefuroxime (Table 2).

This is worrisome knowing that clinicians prescribe Ciprofloxacin frequently for treatment of UTI especially as empirical therapy because of its qualities. However, the widespread use and more often the misuse of antimicrobial drugs in Nigeria and the serious flow of fake drugs in the country's drug market despite all efforts by NAFDAC to control them, has led to a general rise in the resistant strains of bacteria particularly to this drug of choice - Ciprofloxacin. Higher resistance was reported in the USA to Ampicillin and Cotrimoxazole $^{17}$. In Palestine, one study showed a high resistance rate for uropathegens like Pseudomonas aeruginonas, Acinotobacter haemolyticus and Staph saprophyticus with a range of $20-28 \%$ for Ciprofloxacin $\frac{a e r u s}{18}$

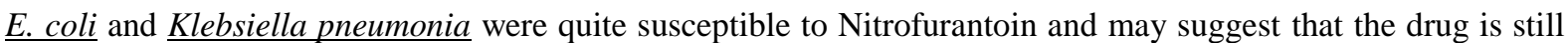
useful for the treatment of simple and uncomplicated UTI if caused by these organisms. Generally, for Ciprofloxacin, higher resistance results were recorded for many uropathogens when compared with studies in other places ${ }^{6,8}$, though these countries are more developed and may have more control in drug use reputation and also in monitoring any imported drugs than what obtains here in Nigeria.

This finding which is not a welcome one, may suggest inappropriate or even non prescription of drugs, for many patients work into patent medicine stores and prescribe and buy drugs freely off-shelf, many of which have doubtful qualities. Many drugs have also faked expiry dates to still enable sellers to commercially dispose of their long expired drugs. It also points at poor infection control strategies in the area. Many do not also perform the necessary laboratory tests or, when they do, do it in ill-equipped laboratories where the tests may be cheaper for the poor quality results.

Conclusively, the results from this study demonstrates a high resistance to many commonly used antimicrobial drugs in our environment, regrettably to Ciprofloxacin which has been widely used for treating UTI in the area and even other numerous infections especially empirically. It therefore suggests the need for a continuous scrutiny and evaluation of the commonly used antimicrobial drugs used in treatment in Nigeria. It therefore recommends for a further step-up for agencies monitoring laboratories and drug importation, manufacture and sales in the area.

Table 1

Distribution of Bacterial Isolates according to Sex

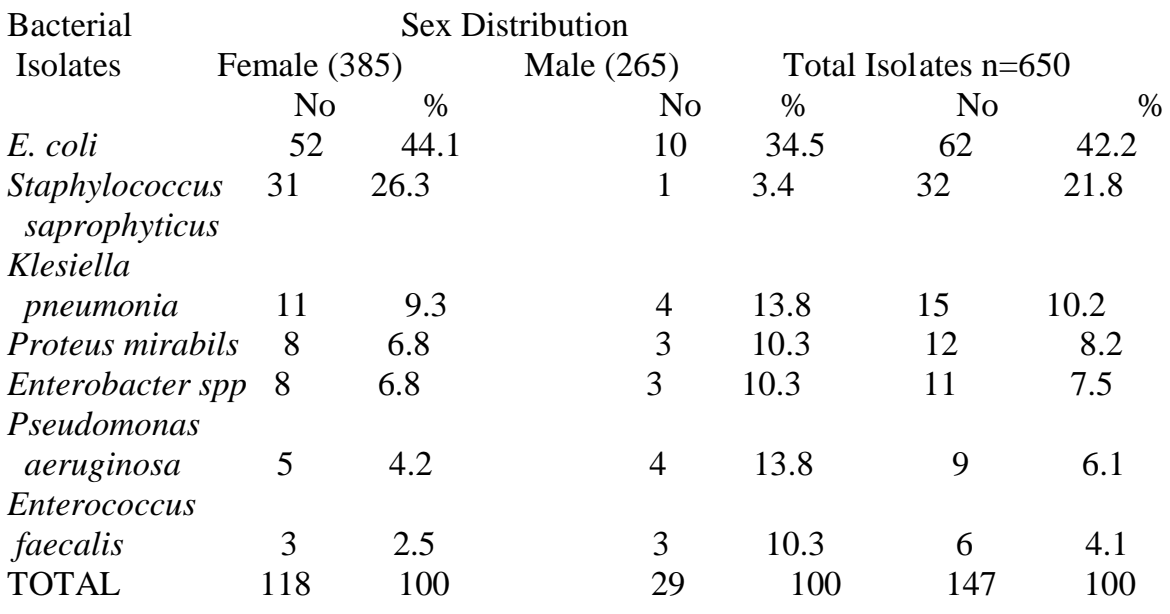

No - Number reflects the number of isolates

Table 2

Percentage of resistance to selected antibiotics for different bacterial isolates

\section{BACTERIA ISOLATES}

1. E. coli

(62)
2. S. saprophyticus
(32)
3. Klebsiella

Antibiotics tested

$\begin{array}{ccccccccc}\text { CIP } & \text { CTX } & \text { STX } & \text { GM } & \text { NTR } & \text { NA } & \text { AM } & \text { CTZ } & \text { CPH } \\ 16.1 & 12 & 60.7 & 19 & 4 & 21 & 83 & 7 & 23\end{array}$

$\begin{array}{lllllllll}30.6 & 25 & 75 & 48.4 & 25 & 100 & 79.6 & 30.6 & 31.3\end{array}$


